\title{
PLÜCKER FORMULAE FOR THE ORTHOGONAL GROUP
}

\author{
KICHOON YANG
}

\begin{abstract}
Plücker formulae for horizontal curves in $S O(\mathrm{~m})$-flag manifolds are derived. These formuJae are seen to generalise the usual Plücker formulae for projective space curves. They also have applications in the theory of minimal surfaces in Euclidean sphere and the complex hyperquadric.
\end{abstract}

\section{INTRODUCTION}

Plücker formulae for projective space curves give relationships between an intrinsic invariant (the genus) and a set of extrinsic invariants (associated degrees and ramification indices). On the other hand the method of moving frames allows us to think of projective curves (whether compact or not) as horizontal curves in the complex flag manifold $S U(n+1) / T$. This gives a new interpretation of the Plücker formulae and indeed one can derive the formulae for horizontal curves in any $G / T$, where $G$ is a connected compact simple Lie group, and $T$ a maximal torus in $G .(G / T$ is called the G-flag manifold.) We note that the resulting formulae have interesting applications to the theory of harmonic maps of real surfaces into Riemannian homogeneous spaces, especially type 1 inner symmetric spaces (see [1, Section 4]).

In this paper we derive explicilly the formulae for horizontal curves in $S O(m) / T$. We do this for compact curves so as to obtain directly the integrated form of the formulae. The cases of $G=S U(n), S p(n)$ have been dealt with in $[4,5]$. It would also be interesting to write down the exceptional Plücker formulae.

\section{Horizontal CURVES in $G$-FLAG Manifolds}

In this section we give an overview of the theory of horizontal curves in $G$-flag manifolds and indicate how the Plücker formulae are derived in general. It should be pointed out that the derivation depends essentially only upon the Maurer-Cartan structure equations of $G$. Horizontal curves form a small subset of a large class of maps called pseudocomplex maps (they are maps from an almost complex manifold to a homogeneous space of the form $G / H$, where $G$ is a compact semisimple Lie group and $H$ is a closed subgroup of maximal rank) and a detailed account of them is given in [4]. In particular the proofs of results collected in this section can be found there.

Received 9 January 1989

Copyright Clearance Centre, Inc. Serial-fee code: 0004.9729/89 \$A2.00+0.00. 
As a motivational example we give a brief discussion of projective curves (see [3, Chapter II]). Consider a projective algebraic curve, that is, a holomorphic map

$$
f=f_{0}: M \rightarrow C P^{n}=S U(n+1) / S(U(1) \times U(n)),
$$

where $M=M_{g}$ is a compact Riemann surface of genus $g$. There are the associated curves

$$
f_{i}: M \rightarrow C G_{n+1, i+1} \subset C P^{N}
$$

where $N=\left(\begin{array}{c}N+1 \\ i+1\end{array}\right)-1,0 \leqslant i \leqslant n-1$. Let $d_{i}$ denote the degree of $f_{i}(M) \subset C P^{N}$ as an algebraic curve and also let $\#_{i}$ denote the total ramification index of $f_{i}$. We then have

$$
2 g-2-\#_{i}=d_{i-1}-2 d_{i}+d_{i+1}, 0 \leqslant i \leqslant n-1,
$$

where $d_{-1}=d_{n}=0$. Let $d s_{N}^{2}$ denote the normalised Fubini-Study metric on $C P^{N}$. Assume that $f$ is nondegenerate, that is $f(M)$ does not lie in a lower dimensional projective subspace. Then each $f_{i}^{*} d s_{N}^{2}$ is a singular metric (also called a pseudometric, for a definition see $[4, \mathrm{p} .81])$ on $M$ and can be written as $\varphi_{i} \bar{\varphi}_{i}$ for some local type $(1,0)$ form $\varphi_{i}$ on $M$. The zeros of $\varphi_{i}$ (which are globally defined) define an integral divisor on $M$ and its degree equals $\#_{i}$.

Now condiser $S U(n+1) / T$, where $T=S\left(U(1)^{n+1}\right)$ is a maximal torus. Fix an invariant complex structure on $S U(n+1) / T$. This amount to fixing a system of positive roots of $S U(n+1)$ relative to $T$. There are exactly $n$ simple roots of $S U(n+1)$ and the corresponding root spaces generate a rank $n$ holomorphic subbundle, called the horizontal bundle, of the holomorphic tangent bundle of $S U(n+1) / T$. The inclusion $T \subseteq H=(S U(1) \times U(n))$ induces the projection $\pi: S U(n+1) / T \rightarrow C P^{n}$. On $C P^{n}$ we use the complex structure coming from the one on $S U(n+1) / T$ so that $\pi$ is holomorphic. We then have

Theorem. Let $f: M \rightarrow C P^{n}$ be a nondegenerate holomorphic curve. Then its Frenet lifting gives rise to a holomorphic horizontal (tangential to the horizontal bundle) curve $\Phi_{f}: M \rightarrow S U(n+1) / T$ which is nondegenerate in the sense that the image does not lie in any $G^{\prime} / T \cap G^{\prime}$, with $G^{\prime}$ a closed subgroup of lower rank. Conversely, given a. holomorphic horizontal nondegenerate curve $F: M \rightarrow S U(n+1) / T$ the composition $\pi \circ F$ is a nondegenerate holomorphic curve in $C P^{n}$.

Proof: See $[4$, p.84-85] for the proof.

Hereafter, $G$ will denote a compact connected simple Lie group of rank $n$ and, $T$ a maximal torus in $G$. By a horizontal curve in $G / T$ we will mean a holomorphic horizontal map $f: M \rightarrow G / T$, where $M=M_{g}$ is a compact Riemann surface of genus 
$g$. We say $f$ is nondegenerale if $f(M)$ does not lie in any $G^{\prime} / T \cap G^{\prime}$ for $G^{\prime}$, a closed subgroup of lower rank.

Consider a nondegenerate horizontal curve $f: M \rightarrow G / T$. Let $\mathcal{H} \subseteq T^{1,0}(G / T) \rightarrow$ $G / T$ denote the horizontal bundle over $G / T$. $\mathcal{H}$ decomposes as

$$
\mathcal{H}=L_{1} \oplus \ldots \oplus L_{n},
$$

where $L_{i} \rightarrow G / T$ is the holomorphic line bundle generated by the root space corresponding to the $i$-th simple root (Order the set of simple roots.) On $G / T$ we fix an invariant metric $d s_{G / T}^{2}$. Let

$$
\langle,\rangle_{i}=\text { the restriction of } d s_{G / T}^{2} \text { to } L_{i} \text {. }
$$

The nondegeneracy assumption of $f$ implies that $f_{i}^{*}($,$) is a singular metric on M$ for every $i$. Put $f_{i}^{*}()=,\varphi_{i} \bar{\varphi}_{i}$, for some local type $(1,0)$ form $\varphi_{i}$ on $M$. Let $D_{i}$ denote the zero divisor of $\varphi_{i}$ and put $\#_{i}=\operatorname{deg} D_{i}$. Also let $\Lambda_{i}$ denote the Kähler form of $\left(M, \varphi \bar{\varphi}_{i}\right) . \Lambda_{i}$ defined on the support of $\varphi_{i} \bar{\varphi}_{i}$, hence on all of $M$ except for a finite set. Put $d_{i}=\int_{M} \Lambda_{i}, 1 \leqslant i \leqslant n$. So $d_{i}$ is the area of $\left(M, \varphi_{i} \bar{\varphi}_{i}\right)$. Observe that if $G=S U(n+1)$ then $d_{i}$ is the degree of the $i$-th associated surve of $f$ by Wirtinger's theorem.

We now indicate how the general derivation of Plücker formulae goes. Choose a $G$-frame $e: U \subset M \rightarrow G, \pi \circ e=f, \pi: G \rightarrow G / T$. By its very construction we can write $\varphi_{i}$ as $e^{*} \Omega_{i}$, where $\Omega_{i}$ is a left-invariant form on $G$. Now

$$
d \varphi_{i}=-\omega_{i} \wedge \varphi_{i}
$$

where $\omega_{i}$ is the complex connection form. On the other hand

$$
d \varphi_{i}=d e^{*} \Omega_{i}=e^{*} d \Omega_{i}
$$

which we can compute using the Maurer-Cartan structure equations of $G$. This allows us to write $\omega_{i}$ as the pullback of left-invariant forms. Exterior differentiation of both sides of the equations thus obtained gives relations amongst the curvature forms, the Gaussian curvatures, and the Kähler forms, which give the infinitesimal form of the Plücker formulae. Integration over $M$ finally yields the formulae.

Remarks. Combining the above theorem on projective curves with the well-known result that any compact Riemann surface can be holomorphically embedded in $C P^{3}$ we can show that any compact Riemann surface can be holomorphically and horizontally embedded in $S U(4) / T$. In light of this it would be interesting to establish the following conjecture: any compact Riemann surface can be holomorphically and horizontally embedded in $G / T$, where $G$ is any connected compact simple lie group of rank 3 . Another interesting problem is to describe the structure of the moduli of horizontal curves. It seems that the set of compact horizontal curves in any $G / T$ forms at least a complex analytic space. 


\section{Plücker Formulae for $S O(2 n)$}

Let $G=S O(2 n)=\left\{\left.X \in G L(2 n ; R)\right|^{t} X X=1\right.$, det $\left.X=1\right\}$ and also let $T=\left\{\operatorname{diag}\left(D_{1}, \ldots, D_{n}\right) \mid D_{i}=\left(\begin{array}{c}c,-s \\ s, c\end{array}\right)\right.$, where $\left.c=\cos 2 \pi x_{i}, s=\sin 2 \pi x_{i}\right\}=S O(2)^{n}$. $T$ is a maxximal torus in $G$ and

$$
G / T=\bar{F}_{2,4, \ldots, 2 n}\left(\mathbf{R}^{2 n}\right)
$$

the full oriented even flags in $\mathbf{R}^{2 n}$.

Notation. We will identify $S O(2)$ with $U(1)$ and write $e^{i x}$ (or exp (ix)) instead of $\left(\begin{array}{c}\cos x,-\sin x \\ \sin x, \cos x\end{array}\right)$ from now on.

We note that the standard injection $U(n) \rightarrow S O(2 n)$ projects down to a totally geodesic embedding $U(n) / U(1)^{n} \rightarrow G / T$. For this reason one should consider the study of horizontal maps in $S O(2 n) / S O(2)^{n}$ to be more general in scope than that in the complex flag manifold.

There is the Lie algebra decomposition $\mathbf{g}=\mathbf{t} \oplus \mathbf{m}$, where $\mathbf{m}$ is the orthogonal vector space complement of $t$ (the Lie algebra of $T$ ) with respect to the Killing form.

Let $E_{i j}=\left[e_{m n}\right], F_{i j}=\left[f_{m n}\right], E_{i j}^{\prime}=\left[e_{m n}^{\prime}\right]$ and $F_{i j}^{\prime}=\left[f_{m n}^{\prime}\right]$ be $2 n \times 2 n$ matrices with all entries zero except for

$$
\begin{aligned}
& e_{2 i-1,2 j-1}=e_{2 i, 2 j}=-e_{2 j-1,2 i-1}=-e_{2 j, 2 i}=1 \\
& -f_{2 i-1,2 j}=f_{2 i, 2 j-1}=-f_{2 j-1,2 i}=f_{2 j, 2 i-1}=1 \\
& e_{2 i-1,2 j-1}^{\prime}=-e_{2 i, 2 j}^{\prime}=-e_{2 j-1,2 i-1}^{\prime}=e_{2 j, 2 i}^{\prime}=1 \\
& f_{2 i-1,2 j}^{\prime}=f_{2 i, 2 j-1}^{\prime}=-f_{2 j-1,2 i}^{\prime}=-f_{2 j, 2 i-1}^{\prime}=1
\end{aligned}
$$

Then

$$
m=\sum V_{i j} \oplus V_{i j}^{\prime}(1 \leqslant i<j \leqslant n),
$$

where $V_{i j}=\mathbf{R}-\operatorname{span}\left\{E_{i j}, F_{i j}\right\}$ and $V_{i j}^{\prime}=\mathbf{R}-\operatorname{span}\left\{E_{i j}^{\prime}, F_{i j}^{\prime}\right\}$.

For $t=\operatorname{diag}\left(D_{1}, \ldots, D_{n}\right) \in T, v=x E_{i j}+y F_{i j} \in V_{i j}$ and $v^{\prime}=x E_{i j}^{\prime}+y F_{i j}^{\prime} \in V_{i j}^{\prime}$ we compute that

$$
\begin{gathered}
A d_{t}: v \leftrightarrow x+i y \mapsto \exp \left(2 \pi i\left(x_{i}-x_{j}\right)\right) \cdot(x+i y), \\
v^{\prime} \leftrightarrow x+i y \mapsto \exp \left(2 \pi i\left(x_{i}+x_{j}\right)\right) \cdot(x+i y),
\end{gathered}
$$

where we use the complex notation to write $v, v^{\prime}$ relative to their respective bases. It follows that the root spaces of $G$ are $V_{i j}, V_{i j}^{\prime}$ and the corresponding roots are $\Delta=\left\{ \pm\left(x_{i}-x_{j}\right), \pm\left(x_{i}+x_{j}\right) \mid 1 \leqslant i<j \leqslant n\right\}$.

Put $\theta_{i j}=x_{i}-x_{j}, \theta_{i j}^{\prime}=x_{i}+x_{j}$. 
Write $\Delta=\Delta_{+} \cup \Delta_{-}$, where $\Delta_{+}=\left\{\theta_{i j}, \theta_{i j}^{\prime}: 1 \leqslant i<j \leqslant n\right\} . \Delta_{+}$forms a system of positive roots and the resulting simple roots are

$$
\Delta_{s}=\left\{\theta_{12}, \theta_{23}, \ldots, \theta_{n-1, n}, \theta_{n-1, n}^{\prime}\right\}
$$

$\Omega=\left(\Omega_{\beta}^{\alpha}\right), 1 \leqslant \alpha, \beta \leqslant 2 n$, denotes the $o(2 n)$-valued Maurer-Cartan form of $G$. The Maurer-Cartan structure equations are

$$
d \Omega_{\beta}^{\alpha}=-\Omega_{\gamma}^{\alpha} \wedge \Omega_{\beta}^{\gamma}
$$

Let $J$ denote the invariant complex structure on $G / T$ arising from the choice, $\Delta_{+}$, of positive roots. For $1 \leqslant i<j \leqslant n$, put

$$
\begin{aligned}
& \Theta^{i j}=\frac{1}{2}\left[\left(\Omega_{2 j-1}^{2 i-1}+\Omega_{2 j}^{2 i}\right)+i\left(\Omega_{2 j-1}^{2 i}-\Omega_{2 j}^{2 i-1}\right)\right], \\
& \Theta^{i j}=\frac{1}{2}\left[\left(\Omega_{2 j-1}^{2 i-1}-\Omega_{2 j}^{2 i}\right)+i\left(\Omega_{2 j-1}^{2 i}+\Omega_{2 j}^{2 i-1}\right)\right] .
\end{aligned}
$$

The type $(1,0)$ forms on $G / T$ are given by $C$-linear combinations of the pullbacks of $\Theta^{i j}, \Theta^{\prime i j}$.

For an invariant metric on $G / T$ we take the pullback of

$$
\frac{1}{2} \sum\left(\Omega_{2 j-1}^{2 i-1}\right)^{2}+\left(\Omega_{2 j}^{2 i}\right)^{2}+\left(\Omega_{2 j-1}^{2 i}\right)^{2}+\left(\Omega_{2 j}^{2 i-1}\right)^{2}, 1 \leqslant i<j \leqslant n
$$

$d s_{G / T}^{2}$ will denote the above metric.

The horizontal bundle $\mathcal{H} \subset T^{1,0}(G / T)$ is given by the following Pfaffian system on $G / T$ :

$$
\sum=\left\{\theta^{i j}=0 \text { unless } j=i+1 ; \theta^{\prime i j}=0 \text { unless }(i, j)=(n-1, n)\right\}
$$

where $\theta^{i j}=e^{*} \Theta^{i j}$ and $e$ is a local section of $G \rightarrow G / T$, etcetera. Write

$$
\mathcal{H}=L_{1} \oplus \ldots \dot{\oplus} L_{n}
$$

where for $1 \leqslant i \leqslant n-1 \quad L_{i}$ is the holomorphic line bundle over $G / T$ generated by the root space $V_{i, i+1}$ and $L_{n}$ is the line bundle generated by $V_{n-1, n}^{\prime}$.

Consider a horizontal curve (that is, horizontal holomorphic map from a compact Riemann surface of genus $g$ ) $f: M \rightarrow G / T$. Let $e: U \subset M \rightarrow G$ be a local lifting of $f$. The holomorphy of $f$ is reflected by the fact that the forms $e^{*} \Theta^{i j}, e^{*} \Theta^{\prime i j}$ are all of type $(1,0)$ on $M$. 
Notation. $e^{*} \Theta^{i j}=\theta^{i j}, e^{*} \Omega_{\beta}^{\alpha}=\omega_{\beta}^{\alpha}$.

The horizontality of $f$ implies that

$$
\left\{\theta^{i j}=0 \text { for } j \neq i+1 ; \theta^{\prime i j}=0 \text { for }(i, j) \neq(n-1, n)\right\} \text {. }
$$

So the surviving type $(1,0)$ forms on $M$ are

$$
\varphi_{i}=\theta^{i, i+1}, 1 \leqslant i \leqslant n-1, \text { and } \varphi_{n}=\theta^{\prime n-1, n}
$$

As in Section 1 let $\langle,\rangle_{i}$ denote $d s_{G / T}^{2}$ restricted to the line bundle $L_{i}$. Then we have

$$
f^{*}(,\rangle_{i}=\varphi_{i} \bar{\varphi}_{i}, 1 \leqslant i \leqslant n
$$

Each $\varphi_{i} \bar{\varphi}_{i}$ defines a singular metric on $M$. In particular, $\varphi_{i}$ has only isolated zeros of finite multiplicity. ( $f$ is nondegenerate if and only if none of the $\varphi_{i}$ 's is identically zero. See $[4$, p.64 and p.76]). Put

$$
\begin{gathered}
D_{i}=\text { the zero divisor of } \varphi_{i} \text { on } M, \\
\#_{i}=\operatorname{deg} D_{i} .
\end{gathered}
$$

Away from the zeros of $\varphi_{i}$ we have

$$
\begin{gathered}
d \varphi_{i}=-\omega_{i} \wedge \varphi_{i} \\
d \omega_{i}=\frac{1}{2} K_{i} \varphi_{i} \wedge \bar{\varphi}_{i}=\left(-i K_{i}\right) \cdot \Lambda_{i}
\end{gathered}
$$

where $\omega_{i}$ is the complex connection form, $d \omega_{i}$ is the curvature form, $K_{i}$ is the Gaussian curvature, and $\Lambda_{i}$ denotes the Kähler form of $\left(M, \varphi_{i} \bar{\varphi}_{i}\right)$.

Differentiating both sides of the equations in (1) we obtain

$$
\begin{gathered}
\omega_{2 j-1}^{2 i-1}=\omega_{2 j}^{2 i}=\omega_{2 j-1}^{2 i}=\omega_{2 j}^{2 i-1}=0, j \neq i+1,1 \leqslant i \leqslant n-2, \\
\omega_{2 i+1}^{2 i-1}=\omega_{2 i+2}^{2 i}, \omega_{2 i+1}^{2 i}=-\omega_{2 i+2}^{2 i-1}, 1 \leqslant i \leqslant n-2 .
\end{gathered}
$$

Using (5) we can rewrite the equations in (2) as follows.

$$
\begin{gathered}
\varphi_{i}=\omega_{2 i+1}^{2 i-1}+i \omega_{2 i+1}^{2 i}, 1 \leqslant i \leqslant n-2, \\
\varphi_{n-1}=\frac{1}{2}\left[\left(\omega_{2 n-1}^{2 n-3}+\omega_{2 n}^{2 n-2}\right)+i\left(\omega_{2 n-1}^{2 n-2}-\omega_{2 n}^{2 n-3}\right)\right] \\
\varphi_{n}=\frac{1}{2}\left[\left(\omega_{2 n-1}^{2 n-3}-\omega_{2 n}^{2 n-2}\right)+i\left(\omega_{2 n-1}^{2 n-2}+\omega_{2 n}^{2 n-3}\right)\right]
\end{gathered}
$$


Using (3), (6) and the Maurer-Cartan structure equations we differentiate both sides of the equations in (2) and obtain

$$
\begin{gathered}
\omega_{i}=-i\left(\omega_{2 i}^{2 i-1}-\omega_{2 i+2}^{2 i+1}\right), 1 \leqslant i \leqslant n-1, \\
\omega_{n}=-i\left(\omega_{2 n-2}^{2 n-3}+\omega_{2 n}^{2 n-1}\right) .
\end{gathered}
$$

Using the Maurer-Cartan structure equations, (2), and the definition of the $\Lambda_{i}$ 's we also obtain

$$
\begin{gathered}
d \omega_{2 i}^{2 i-1}=-2 \Lambda_{i-1}+2 \Lambda_{i}, 1 \leqslant i \leqslant n-2, \Lambda_{0}=0 \\
d \omega_{2 n-2}^{2 n-3}=-2 \Lambda_{n-2}+2\left(\Lambda_{n-1}+\Lambda_{n}\right) \\
d \omega_{2 n-1}^{2 n-1}=-2 \Lambda_{n-1}+2 \Lambda_{n} .
\end{gathered}
$$

Exterior differentiation of the equations in (7), using (8) together with (4), now gives

$$
\begin{gathered}
K_{i} \Lambda_{i}=-2 \Lambda_{i-1}+4 \Lambda_{i}-2 \Lambda_{i+1}, 1 \leqslant i \leqslant n-3, \Lambda_{0}=0 \\
K_{n-2} \Lambda_{n-2}=-2 \Lambda_{n-3}+4 \Lambda_{n-2}-2 \Lambda_{n-1}-2 \Lambda_{n} \\
K_{n-1} \Lambda_{n-1}=-2 \Lambda_{n-2}+4 \Lambda_{n-1}, \\
K_{n} \Lambda_{n}=-2 \Lambda_{n-2}+4 \Lambda_{n},
\end{gathered}
$$

where we assume $G=S O(2 n)$ with $n \geqslant 5$.

For $n=4$ we get

$$
\begin{gathered}
K_{1} \Lambda_{1}=4 \Lambda_{1}-2 \Lambda_{2} \\
K_{2} \Lambda_{2}=-2 \Lambda_{1}+4 \Lambda_{2}-2 \Lambda_{3}-2 \Lambda_{4} \\
K_{3} \Lambda_{3}=-2 \Lambda_{2}+4 \Lambda_{3} \\
K_{4} \Lambda_{4}=-2 \Lambda_{2}+4 \Lambda_{4}
\end{gathered}
$$

For $n=3$ we get

$$
\begin{gathered}
K_{1} \Lambda_{1}=4 \Lambda_{1}-2 \Lambda_{2}-2 \Lambda_{3} \\
K_{2} \Lambda_{2}=-2 \Lambda_{1}+4 \Lambda_{2} \\
K_{3} \Lambda_{3}=-2 \Lambda_{1}+4 \Lambda_{3} .
\end{gathered}
$$

Finally for $n=2$,

$$
\begin{aligned}
& K_{1} \Lambda_{1}=4 \Lambda_{1} \\
& K_{2} \Lambda_{2}=4 \Lambda_{2}
\end{aligned}
$$


The generalised Gauss-Bonnet-Chern theorem $([3$, p.144] or $[4$, p.81]) states that

$$
\int_{M} d \omega_{i}=2 \pi i \cdot\left(2 g-2-\#_{i}\right)
$$

where $g$ is the genus of $M$. This allows us to integrate the left hand sides of the equations in (9). Also put

$$
d_{i}=\frac{1}{\pi} \int_{M} \Lambda_{i}=\text { the area of }\left(M, \varphi_{i} \bar{\varphi}_{i}\right) .
$$

Upon integration over $M$ the equations in (9) yields

$$
\begin{gathered}
2 g-2-\#_{i}=d_{i-1}-2 d_{i}+2 d_{i+1}, 1 \leqslant i \leqslant n-3, d_{0}=0, \\
2 g-2-\#_{n-2}=d_{n-3}-2 d_{n-2}+d_{n-1}+d_{n}, \\
2 g-2-\#_{n-1}=d_{n-2}-2 d_{n-1}, \\
2 g-2-\#_{n}=d_{n-2}-2 d_{n} .
\end{gathered}
$$

\section{Plücker formulae for $S O(2 n+1)$}

For the rest of the paper we let $G=S O(2 n+1)$. Include $S O(2 n)$ in $S O(2 n+1)$ via $g \mapsto\left(\begin{array}{l}g, 0 \\ 0,1\end{array}\right)$. For a maximal torus in $G$ we use $T$ in Section 2 included in $G$ which we again denote by $T$. Generally speaking we will identify an object in $S O(2 n)$ (or in $o(2 n))$ with its counterpart in $S O(2 n+1)$ (or in o $(2 n+1))$.

As the root structure of $S O(2 n+1)$ is different from that of $S O(2 n)$ it is necessary that we treat this case separately. Nevertheless the computations involved are similar and will mostly be suppressed.

As before we have the Lie algebra decomposition $\mathbf{g}=\mathbf{t} \oplus \mathbf{m}, \mathbf{m}=\mathbf{t}^{\perp}$. Let $E_{i j}$, $F_{i j}, E_{i j}^{\prime}, F_{i j}^{\prime}$ be as in Section 2 except that they are now interpreted to be vectors in $\mathbf{g}=\mathbf{o}(2 n+1)$. Let $E_{\gamma}=\left[e_{i k}\right]$ be the $(2 n+1) \times(2 n+1)$ matrix with all entries zero except for $e_{\gamma, 2 n+1}=-e_{2 n+1, \gamma}=1$. Then

$$
m=\sum V_{i j} \oplus V_{i j}^{\prime} \oplus V_{i}=o(2 n) \oplus \sum V_{i}(1 \leqslant i<j \leqslant n, 1 \leqslant i \leqslant n),
$$

where $V_{i}=\mathbf{R}$-span $\left\{E_{2 i-1}, E_{2 i}\right\}$ and $V_{i j}, V_{i j}^{\prime}$ as before.

For $t=\operatorname{diag}\left(D_{1}, \ldots, D_{n}, 1\right) \in T$ and $v=x E_{2 i-1}+y E_{2 i}=\left(\begin{array}{l}x \\ y\end{array}\right)$ we compute that

$$
A d_{t}: v \leftrightarrow x+i y \rightarrow \exp \left(2 \pi i x_{i}\right) \cdot(x+i y) .
$$

'This together with the earlier computation shows that $V_{i j}, V_{i j}^{\prime}, V_{i}$ are the root spaces of $G$ relative to $T$. The roots are

$$
\Delta=\left\{ \pm\left(x_{i}-x_{j}\right), \pm\left(x_{i}+x_{j}\right), \pm x_{i}: 1 \leqslant i<j \leqslant n\right\} \text {. }
$$


Put $\theta_{i j}=x_{i}-x_{j}, \theta_{i j}^{\prime}=x_{i}+x_{j}, \theta_{i}=x_{i}$.

For positive roots we take $\Delta_{+}=\left\{\theta_{i j}, \theta_{i j}^{\prime}, \theta_{i}: 1 \leqslant i<j \leqslant n\right\}$. The resulting simple roots are $\Delta_{s}=\left\{\theta_{12}, \theta_{23}, \ldots, \theta_{n-1, n}, \theta_{n}\right\}$.

$\Omega=\left(\Omega_{\beta}^{\alpha}\right), 1 \leqslant \alpha, \beta \leqslant 2 n+1$, denotes the $o(2 n+1)$-valued Maurer-Cartan form of $G$. For $1 \leqslant i<j \leqslant n$ put

$$
\begin{gathered}
\Theta^{i j}=\frac{1}{2}\left[\left(\Omega_{2 j-1}^{2 i-1}+\Omega_{2 j}^{2 i}\right)+i\left(\Omega_{2 j-1}^{2 i}-\Omega_{2 j}^{2 i-1}\right)\right] \\
\Theta^{\prime i j}=\frac{1}{2}\left[\left(\Omega_{2 j-1}^{2 i-1}-\Omega_{2 j}^{2 i}\right)+i\left(\Omega_{2 j-1}^{2 i}+\Omega_{2 j}^{2 i-1}\right)\right] \\
\Theta^{i}=\Omega_{2 n+1}^{2 i-1}+i \Omega_{2 n+1}^{2 i}
\end{gathered}
$$

'The pullbacks of $\Theta^{i j}, \Theta^{\prime i j}, \Theta^{i}$ to $G / T$ are all of type $(1,0)$ with respect to the invariant complex structure $J$ determined by $\Delta_{+}$.

For an invariant metric on $G / T$ we take the pullback of

$$
\frac{1}{2} \sum\left[\left(\Omega_{2 j+1}^{2 i-1}\right)^{2}+\left(\Omega_{2 j}^{2 i}\right)^{2}+\left(\Omega_{2 j-1}^{2 i}\right)^{2}+\left(\Omega_{2 j}^{2 i-1}\right)^{2}+2\left(\Omega_{2 n+1}^{2 i-1}\right)^{2}+2\left(\Omega_{2 n+1}^{2 i}\right)^{2}\right]
$$

We consider a horizontal curve $f: M \rightarrow G / T$. Letting $e: U \subseteq M \rightarrow G$ be a local lifting of $f$ we write $e^{*} \Omega=\omega, e^{*} \Theta=\theta$. Then the horizontality of $f$ implies that

$$
\left\{\theta^{i j}=0 \text { unless } j=i+1 ; \theta^{i j} \text { for } 1 \leqslant i<j \leqslant n ; \theta^{i}=0 \text { unless } i=n\right\} \text {. }
$$

The surviving type $(1,0)$ forms on $M$ are

$$
\varphi_{i}=\theta^{i, i+1}, 1 \leqslant i \leqslant n-1 ; \varphi_{n}=\theta^{n}=\omega_{2 n+1}^{2 n-1}+i \omega_{2 n+1}^{2 n} .
$$

As before put

$$
\begin{gathered}
\#_{i}=\text { the degree of the zero divisor of } \varphi_{i}, \\
\qquad \begin{array}{c}
d_{i}=\frac{1}{\pi} \int_{M} \Lambda_{i} \text { for } 1 \leqslant i \leqslant n-1, \\
d_{n}=\frac{1}{2 \pi} \int_{M} \Lambda_{n},
\end{array}
\end{gathered}
$$

where $\Lambda_{i}(1 \leqslant i \leqslant n)$ denotes the Kähler form of $\left(M, \varphi_{i} \bar{\varphi}_{i}\right)$. Going through a derivation similar to the one already given in Section 2 we obtain

$$
2 g-2-\#_{i}=d_{i-1}-2 d_{i}+d_{i+1}, 1 \leqslant i \leqslant n,
$$

where $d_{0}=0, d_{n+1}=d_{n}$. 
Remark. Let $H=S O(2) \times S O(2 n-2), G / H=Q_{2 n-2} \subset C P^{2 n-1}$, the hyperquadric. An inclusion $T \subset H$ induces a projection $G / T \rightarrow G / H$. Then a horizontal curve in $G / T$ projects down to a harmonic map into $G / H$ by Bryant's theorem. Also if $G=S O(2 n+1)$ then we can take $H=S O(2 n)$ and produce harmonic maps into $S^{2 n}=G / H$ from horizontal curves. It is not difficult to show that every minimal twosphere in $S^{2 n}$ arises as a horizontal curve. Thus our formulae in this section represent a generalisation of the similar formulae given by Chern in [2].

\section{REFERENCES}

[1] R.L. Bryant, 'Lie groups and twistor space', Duke Math. J. 62 (1985), 223-262.

[2] S.S. Chern, 'On the minimal immersions of the two-sphere in a space of constant curvature', in Problems in Analysis, Editor R.C. Gunning, pp. 27-40 (Princeton, 1970).

[3] P. Griffiths and J. Harris, Principles of Algebraic Geometry (Wiley, New York, 1978).

[4] K. Yang, Almost Complex Homogeneous Spaces and Their Submanifolds (World Scientific Publishing, Singapore-New Jersey, 1987).

[5] K. Yank, 'Horizontal holomorphic curves in SP(n)-flag manifolds', Proc. Amer. Math. Soc. 103 (1988), 265-273.

Department of Mathematics

Arkansas State University

State University, Ar 72467

United States of America 